Cuadernos de Lingüística Hispánica $n^{\circ} .24$ ISSN 0121-053X • ISSN en línea 2346-1829 Julio-Diciembre 2014, pp. 105-122

\title{
Saber constituido al interior de los discursos sobre calidad de educación superior ${ }^{*}$
}

\author{
LUZ ELENA BATANELO GARCÍA**
}

lebatane@ut.edu.co

Recepción: 30 de septiembre de 2013

Aprobación: 25 de noviembre de 2013

Cómo citar este artículo: Batanelo García, L. (2014). Saber constituido al interior de los discursos sobre calidad de educación superior. Cuadernos de Lingüística Hispánica, 24, 105-122. Tunja: Uptc.

\footnotetext{
Artículo de investigación científica.

* Licenciada en Español e Inglés. Magister en Lingüística Española del Instituto Caro y Cuervo, Colombia. Candidata a Doctora en Ciencias de la Educación de RUDECOLOMBIA- Universidad del Tolima. Investigadora del Grupo LINGUA. Docente de Planta de Tiempo Completo en el Área de Pedagogía del Lenguaje de la Facultad en Ciencias de la Educación de la Universidad del Tolima, Colombia.
} 


\title{
Resumen
}

Este estudio del saber constituido al interior de los discursos, en diálogo con autores como Michel Foucault, Jorge Eliécer Martínez Posada y Luís Alfonso Ramírez Peña, abordado desde la investigación Formación y regularidades de los enunciados discursivos de la calidad de la educación superior en Colombia entre 1991 y 2013, describe la coexistencia, sucesión, funcionamiento, determinación recíproca y transformación independiente y correlativa de los enunciados discursivos acerca de la calidad de la educación superior en los ámbitos internacional y nacional. Se explicitan los resultados en el proceso arqueológico propuesto por el método histórico-crítico de Foucault y se concluye que la calidad se visibiliza en un horizonte de globalización económica, aplicación de políticas neoliberales, reforma del Estado, y a través de tareas de inspección, vigilancia y control.

Palabras clave: arqueología, calidad de educación superior, enunciados.

\section{Knowledge in discourses on the quality of higher education quality}

\begin{abstract}
This study of knowledge constituted in discourses, in dialogue with authors such as Michel Foucault, Jorge Eliécer Martínez Posada and Luis Alfonso Ramírez Peña, is based on the research project Training and regularities in the discursive utterances of the quality of higher education in Colombia between 1991 and 2013, and describes the coexistence, succession, functioning, reciprocal determination, independent and correlative transformation of discursive utterances related to the quality of higher education both nationally and internationally. The results are explained in the archaeological process proposed by Foucault's historical-critical method, and it is concluded that quality is visible within a horizon of economic globalization, implementation of neoliberal policies, State reforms, and through works of inspection, supervision and control.
\end{abstract}

Key words: archaeology, quality of higher education, utterances. 


\title{
Le savoir constitué à l'intérieur des discours sur la qualité de l'éducation supérieure
}

\begin{abstract}
Résumé
Cette étude sur le savoir constitué à l'intérieur des discours, en dialogue avec les auteurs comme Michel Foucault, Jorge Eliécer Martínez Posada et Luís Alfonso Ramírez Peña, et abordée à partir de la recherche Formation et régularités des énoncés discursifs de la qualité de l'éducation supérieure en Colombie entre 1991 et 1993, décrit la coexistence, succession, fonctionnement, détermination réciproque et transformation indépendante et corrélative des énoncés discursifs à propos de la qualité de l'éducation supérieure dans les scènes nationale et internationale. On explicite les résultats dans le processus archéologique proposé par la méthode historique-critique de Foucault. Et on conclut que la qualité devient visible dans un horizon de globalisation économique, d'application de politiques néolibérales, de réforme de l'État, et à travers de l'inspection, la vigilance et le contrôle.
\end{abstract}

Mots clés: archéologie, qualité d'éducation supérieure.

\section{Saber constituído ao interior dos discursos sobre qualidade da educação superior}

\section{Resumo}

Este estudo do saber constituído ao interior dos discursos, em diálogo com autores como Michel Foucault, Jorge Eliécer Martínez Posada e Luís Alfonso Ramírez Peña, abordado desde a pesquisa Formação e regularidades dos enunciados discursivos da qualidade da educação superior na Colômbia entre 1991 e 2013, descreve a coexistência, sucessão, funcionamento, determinação recíproca e transformação independente e correlativa dos enunciados discursivos acerca da qualidade da educação superior nos âmbitos internacional e nacional. Explicitam-se os resultados no processo arqueológico proposto pelo método histórico-crítico de Foucault e se conclui que a qualidade se visibiliza em um horizonte de globalização econômica, aplicação de políticas neoliberais, reforma do Estado, e através de tarefas de inspeção, vigilância e controle.

Palavras chave: arqueologia, qualidade da educação superior, enunciados. 


\section{Introducción}

Para Colombia y la comunidad académica interesada en las investigaciones sobre educación superior es de gran interés el estudio desde la primera fase metodológica propuesta por Michel Foucault (1969), con el propósito de comprender ¿Cómo se configura arqueológicamente una interpretación de los enunciados discursivos acerca de la calidad de la Educación Superior en Colombia entre 1991 y 2013? Esta pregunta requiere una reflexión del campo discursivo y la descripción de los hechos, en donde es necesario, primero, captar el enunciado acerca de la calidad de la educación superior; y segundo, estudiar la calidad de la educación superior a la manera de acontecimiento enunciativo para no encasillarla en intenciones del autor, forma de su intelecto, rigor del pensamiento, temas de obsesión y proyecto de existencia, sino poder visibilizar sus regularidades y otros tipos de conexiones. Los dos anteriores procesos son los que se socializan en el presente artículo.

Según Foucault (1969), la calidad de la educación superior se constituye en un sistema de formación discursiva, donde:

No se entiende únicamente la yuxtaposición, la coexistencia o la interacción de elementos heterogéneos (instituciones, técnicas, grupos sociales, organizaciones perceptivas, relaciones entre discursos diversos), sino su entrada en relación $-\mathrm{y}$ bajo una forma bien determinada- por la práctica discursiva (p. 119).

Es un discurso porque es "una práctica regulada que da cuenta de cierto número de enunciados" (p. 132). Para este autor, el enunciado es una función que trasciende el dominio de estructuras y unidades lógicas, gramaticales y de actos de habla. Una función a la que hay que describir en su ejercicio, en sus condiciones, reglas que la controlan y el campo en el que se efectúa. Los enunciados conforman prácticas discursivas "en las que deben referirse los hechos de sucesión, si no se quiere establecerlos de una manera salvaje e ingenua, es decir en términos de mérito” (p. 241). Pero ¿cómo realizar el proceso de interpretación de las prácticas discursivas constituidas por enunciados? 
Al estudiar la calidad de la educación superior, es fundamental realizar una arqueología, ya que esta pone de manifiesto unas relaciones entre las formaciones discursivas y unos dominios no discursivos (instituciones, acontecimientos políticos, prácticas y procesos económicos). Foucault señala que "La arqueología puede así constituir el árbol de derivación de un discurso" (p. 272). Además, define las estructuras observables y el campo de objetos posibles (prescripciones, formas de descripción, códigos perceptibles, posibilidades de caracterización), los cuales construyen dominios de conceptos; constituyen estrategias, tales como transformaciones conceptuales, emergencias de nociones inéditas, fundamentación de técnicas, entre otras. Continúa afirmando que construir una historia de los enunciados es definir los discursos a la manera de prácticas que obedecen unas reglas; realizar un análisis diferencial de las modalidades de discurso; determinar tipos y reglas de prácticas discursivas más allá de la afectación entre lo individual y lo social; es una reescritura de la transformación de lo que ha sido y se ha escrito; es la descripción sistemática de un discurso-objeto.

De otro lado, Foucault propone que el apriori histórico es el conjunto de reglas que caracterizan una práctica discursiva. Es la forma de la positividad desde el campo donde pueden eventualmente desplegarse identidades formales, continuidades temáticas, traslaciones de conceptos, juegos polémicos. El apriori da cuenta de los enunciados en su dispersión, las grietas abiertas por su no coherencia, encabalgamiento, reemplazamiento recíproco, simultaneidad y su sucesión.

Así, el discurso de la calidad de la educación superior no tiene un solo sentido o una verdad, sino una historia, dispersión en el tiempo, modo de sucesión, estabilidad, reactivación, desarrollo y rotación. Una arqueología requiere construir el archivo o sistema general de formación y transformación de los enunciados. El archivo define una práctica que hace surgir multiplicidad de enunciados que se constituyen en acontecimientos. Este hace aparecer las reglas de una práctica que permiten la subsistencia y modificación regular de los enunciados. El archivo posibilita que la arqueología describa los discursos como prácticas especificadas en éstos.

De esta forma, Foucault indica que en el archivo se distinguen los documentos y los monumentos, puesto que:

La historia ha cambiado de posición respecto del documento: se atribuye como tarea primordial, no el interpretarlo, ni tampoco determinar si es veraz y cuál sea su valor expresivo, sino trabajarlo desde el interior y elaborarlo. La historia lo organiza, lo recorta, lo distribuye, lo ordena, lo reparte en niveles, establece series, distingue lo que es pertinente de lo que no lo es, fija elementos, define unidades, describe relaciones" ( p. 10). 
Expone también que la historia transforma los documentos en monumentos cuando adiciona otras relaciones a lo que éste había sido: "despliega una masa de elementos que hay que aislar, agrupar, hacer pertinentes, disponer en relaciones, constituir en conjuntos" (p. 11). Y con la intención de describir los enunciados, prácticas y formaciones discursivas acerca de la calidad de la educación superior ordenadas en una totalidad, abierta a las relaciones de exterioridad y la búsqueda de su origen, se debe:

Emprender la historia de lo que ha sido dicho; rehacer en otro sentido el trabajo de la expresión: remontarse desde los enunciados conservados al hilo del tiempo y dispersados a través del espacio, hacia ese secreto interior que los ha precedido, que se ha depositado en ellos y que en ellos se encuentra traicionado" (p. 205).

\section{Metodología}

El diseño metodológico tiene como base el enfoque con tendencia de significación intersubjetiva y subjetiva propuesto por Michel Foucault frente a las prácticas discursivas, las que se entienden como conjuntos de enunciados que provienen de un mismo sistema de formación y se articulan históricamente en saberes. La arqueología pretende interpretar los documentos internacionales y nacionales sobre calidad de la educación superior, durante los últimos 20 años, para reconocer ¿Cuál es la descripción de coexistencia, sucesión, funcionamiento, determinación recíprocay transformación independiente y correlativa de los enunciados?

Foucault asume el orden arqueológico no desde las sistematicidades ni a partir de las sucesiones cronológicas. Por esta razón, en la visibilización de la historia se asume metodológicamente la construcción del archivo, selección de los enunciados y el análisis de interdependencia enunciativa con el enfoque de Luís Alfonso Ramírez Peña (2006, pp. 8-11), porque posibilita comprender, explicar e interpretar el discurso acerca de la calidad de la educación superior entre 1991 y 2013, a partir de:

- Ubicar el ámbito y género discursivo.

- Identificar las voces en los textos y en el proceso enunciativo: presencia de un "yo" interno del discurso, un "yo" marcador de la situación de comunicación y una sintaxis y articulación de las voces.

- Reconocer los procesos de representación, enunciación y discursivización en las diversas instancias enunciativas.

- Explicar e interpretar desde una perspectiva de un lector-productor de discursos.

En esta publicación, por corresponder a ámbitos internacional y nacional, diversos géneros discursivos y textuales (informes, conferencias, declaraciones, documentos argumentativos, planes de desarrollo y actos legislativos), diferentes voces y modos de 
representar e interrelacionar lo dicho, se toman los enunciados presentes en siete documentos que se encuentran como anexos, con las respectivas citas textuales, en las cuales SE RESALTA CON MAYÚSCULA FIJA las que son bases de la interpretación.

Estos documentos son: el Plan Atcon, la universidad latinoamericana, clave para un enfoque conjunto del desarrollo coordinado social, económico y educativo en América Latina, de R. P Atcon, en 1963 (Cuadro No.1). La Conferencia Mundial sobre Educación para todos. Satisfacción de las necesidades básicas de aprendizaje de Jomtien. Tailandia, 1990 (Cuadro No. 2). La Declaración de Quito. Reunión de ministros de educación de América Latina y el Caribe, en Quito, Ecuador, 1991 (Cuadro No. 3). El documento El desarrollo en la práctica. La enseñanza superior. Las lecciones derivadas de la experiencia, del Banco, desde Washington, D. C., 1994 (Cuadro No. 4). El trabajo Prioridades y Estrategias para la Educación. Examen del Banco Mundial, desde Washington, en 1996 (Cuadro No. 5). La Declaración Mundial sobre la Educación Superior en el Siglo XXI, por parte de la UNESCO, en París, 1998 (Cuadro No. 6). Y el Plan de Desarrollo "La revolución pacífica" Plan de Desarrollo Económico y Social de 1991, desde la Presidencia de la República del Gobierno de César Gaviria (1990-1994), (Cuadro No. 7).

\section{Resultados y su interpretación}

Con los documentos del archivo se evidencia cómo la calidad de la educación superior configura una formación discursiva, a partir de la descripción de los siguientes enunciados: desde lo internacional, en 1963, se dice que "la universidad debe mejorar la calidad de la docencia para conseguir un producto mejor". Luego, con mayor énfasis y en forma progresiva, en 1990 se explicita "la calidad constituida en política de servicio educativo"; en 1991 se establece "el mejoramiento de la calidad como área prioritaria para el crecimiento que requiere medición"; en 1994 se define la "certificación de la calidad mediante la acreditación"; en 1996 se enfatiza en que "la calidad es difícil de definir y medir; por esto está determinada por las condiciones de aprendizaje y por los resultados obtenidos de los estudiantes"; en 1998 se particulariza "la calidad de la enseñanza superior en un concepto pluridimensional que debe comprender todas sus funciones y actividades: enseñanza y programas académicos, investigación y becas, personal, estudiantes, edificios, instalaciones, equipamiento y servicios a la comunidad y al mundo universitario"; y en 1999 se declara que "al Estado le corresponde brindar información transparente sobre calidad y equidad educativa de acuerdo a sistemas de evaluación".

En el ámbito nacional, en 1991 se plantea "elevar la calidad de la educación en todos los niveles, lo cual significa establecer un sistema nacional de evaluación de la calidad"; por lo cual, a partir de 1991 le corresponde al "Estado regular y ejercer la suprema inspección y vigilancia de la educación, con el fin de velar por su calidad"; y se ordena a dicho Estado, a partir de 1992, velar por "la calidad de la educación superior 
dentro del respeto a la autonomía universitaria y a las libertades de enseñanza, aprendizaje, investigación y cátedra", a través de dos sistemas y organismos constituidos para el Colombia desde el Ministerio de Educación Nacional (MEN): el Sistema Nacional de Acreditación para las Instituciones de Educación Superior (el cual desde el 2002 ha sido denominado SACES, Sistema de Aseguramiento de la Calidad para la Educación Superior), el Consejo Nacional de Acreditación (CNA), el Consejo Nacional de Educación Superior (CESU) y el Sistema Nacional de Información de la Educación Superior (SNIES).

De acuerdo con Foucault (1969), la anterior formación discursiva conlleva un sistema de dispersión, regularidad de objetos, tipos de enunciación, conceptos y elecciones temáticas, ordenadas, correlacionadas, con posiciones, funcionamientos y transformaciones. Concibe el objeto de discurso a partir de las condiciones históricas en que se puede "decir de él algo" o las condiciones para que este se inscriba en un dominio de parentesco con otros objetos, o establezca relaciones de semejanza, vecindad, alejamiento, diferencia y transformación: Así, la calidad como objeto de discurso se correlaciona con las funciones de la universidad: calidad en la docencia -la enseñanza-; calidad en la investigación; y calidad en la extensión. En el cuadro ${ }^{1}$ No. 1, del Plan Atcon se relaciona calidad con formas de enseñanza, la cual a partir de 1991 con la Declaración de Quito, cuadro No. 3, se enfoca por la proximidad entre calidad, currículo y propósitos de formación. El Banco Mundial considera la enseñanza superior como algo fundamental para el desarrollo económico y social de los países; desde esta perspectiva, se establece la calidad en relación con la investigación (Cuadro No. 4). Estos cruces de los objetos, desde lo interior hacia el exterior de las Instituciones universitarias, se concretan en 1998 con la Conferencia Mundial sobre Educación Superior, cuando la calidad cubre la última función de extensión y debe dimensionarse y evaluarse internacional y nacionalmente por medio de normas e instancias (Cuadro No. 6).

Foucault (1969) concibe los conceptos en razón de las maneras en que se ordenan las descripciones o los relatos, unidos a las técnicas de reescritura. Los conceptos están ligados a las formas de jerarquía y de subordinación que rigen los enunciados de un texto; también se establecen a partir de los modos de aproximación y de desarrollo de estos enunciados. Los modos de crítica, de comentario y de interpretación de los enunciados formulados constituyen un sistema de formación conceptual. Así, la calidad se asume conceptualmente a partir de la lógica de política de servicio educativo, desde la Conferencia Mundial de Educación para Todos, en 1990, y en forma vertical se impone en el ámbito internacional por organismos constituidos-avalados-legitimados, tales como: UNESCO (Organización de las Naciones Unidas para la Educación, la Ciencia y la Cultura), OREALC (Oficina Regional de Educación de la UNESCO para América Latina y el Caribe), OCDE (Organización de Cooperación y Desarrollo Económico), CEPAL (Comisión económica para

1 En el apartado de anexos que aparece al final del artículo se registra cada uno de los 7 cuadros con el análisis discursivo respectivo. 
América Latina y el Caribe), BANCO MUNDIAL (Banco Internacional de Reconstrucción y Fomento) y BID (Banco Interamericano de Desarrollo).

Toda la configuración anterior se apropia en el ámbito de Colombia por medio de normas o imperativos (leyes, decretos, resoluciones promulgadas por el presidente de la República, el Congreso de Colombia y el Ministerio de Educación Nacional) y aplicada por sistemas y organismos: además de los mencionados: SACES, SNIES, CESU y CNA, están SUE (Sistema de Universidades Estatales), SNCyT (Sistema Nacional de Ciencia y Tecnología), SIG (Sistema Integrado de Gestión de Calidad), ICFES (Instituto Colombiano para el Fomento de la Educación Superior), COLCIENCIAS (Instituto Colombiano para el Desarrollo de la Ciencia y la Tecnología), ICETEX (Instituto Colombiano de Crédito Educativo y Estudios en el Exterior), CNCyt (Consejo Nacional de Ciencia y Tecnología), CONACES (Comisión Nacional Intersectorial para el Aseguramiento de la Calidad de la Educación Superior) y CODECyT (Comisiones Departamentales de Ciencia y Tecnología), entre otros.

Teniendo en cuenta las modalidades enunciativas o aquellos lugares de dispersión; los estatutos, ámbitos, posiciones desde donde se pronuncia un discurso y las discontinuidades de los planes desde los que se habla (Foucault, 1969), la calidad se enuncia como aquello que requiere mejoramiento y/o aseguramiento. Formas de decir que no son sinónimas porque se establecen a partir de intenciones de las instituciones de educación superior desde su autonomía y del Estado en su figura de inspector. La calidad por lo que no es concreta, se plantea con debilidades y carencias, las cuales se deben fortalecer a través de lineamientos, estándares, factores e indicadores. En Colombia, desde 1991, con el Plan de Desarrollo del Gobierno de César Gaviria (Cuadro No. 7) se determina la necesidad de establecer un Sistema Nacional de Evaluación de la Calidad. Este sistema durante la década del 90 y los diez primeros años del 2000 se ha configurado en una estructura que tiene una primera fase que propende hacia el mejoramiento de la calidad, desde la autoevaluación de los programas académicos y las instituciones de educación superior, teniendo en cuenta su autonomía y autorregulación. Y en una segunda etapa, el Estado con sus sistemas y organismos evalúa, registrando, calificando y certificando un aseguramiento de dicha calidad.

Los anteriores pasos posibilitan visibilizar las estrategias discursivas o maneras sistemáticas de tratar los objetos de discurso, delimitándolos, reagrupándolos, separándolos, encadenándolos o derivándolos. Estas formas de enunciación, reelegidas, situadas, constituidas por series, compuestas por grandes unidades teóricas, llegan a manipular los conceptos, a través de reglas de utilización, por coherencias y arquitecturas conceptuales (Foucault, 1969). A partir de estas estrategias que llevan implícitas una racionalidad discursiva, la calidad es singularmente enunciada desde un diseño conceptual: en relación con la evaluación: configurada por autoevaluación, evaluación externa y evaluación de 
pares, procesos realizados por las instituciones de educación superior (IES) y los organismos estatales CONACES y CNA. Configurada en certificación por medio de actos legislativos de los organismos anteriores: resoluciones que renuevan por períodos de tiempo $(3,507$ años) los registros calificados o verificación del cumplimiento de las condiciones de calidad de los programas académicos. Asumida por la acreditación y el reconocimiento_de otros, por ahora en Colombia, solo por parte del Estado desde el CNA, el cual por medio de resoluciones acredita la alta calidad de los programas académicos y de las Instituciones de Educación superior desde su organización, funcionamiento y cumplimiento de la función social. En el país aún no se ha regulado la acreditación por comunidades: institutos, centros $u$ otras organizaciones nacionales o Internacionales. Y constituida por la medición de resultados, desde el ICFES, organismo colombiano que diseña y aplica pruebas estatales, como las Saber Pro, a los estudiantes de últimos semestres de los programas académicos de las IES.

Siguiendo la anterior formación discursiva, se comprende por qué y para qué la calidad de la educación superior constituye un acontecimiento enunciativo. Foucault distingue varios planos de acontecimientos posibles. El plano de los propios enunciados en su emergencia singular. El plano de la aparición de los objetos, tipos de enunciación, conceptos y elecciones estratégicas. El plano de la derivación de nuevas reglas de formación a partir de las reglas que están ya actuando. Y el plano en el que se efectúa la sustitución de una formación discursiva por otra o aparición y desaparición de una positividad (Foucault, 1969, p. 287).

Desde la Conferencia Mundial sobre Educación para todos, en 1998, la calidad es una condición para la equidad educativa. Pero esta visión, en 1994, con el Banco Mundial se modifica por una enseñanza superior de capital importancia para el desarrollo económico y social; y por consiguiente, la calidad de la enseñanza, la investigación y la extensión en el nivel superior como acontecimiento enunciativo debe responder al crecimiento de las fuerzas del mercado y la demanda global de recursos humanos muy calificados; así se observa en la cita que aparece en el anexo correspondiente al Cuadro No. 5:

Las enormes transformaciones producidas recientemente en los mercados de trabajo por las reformas económicas, la integración de la economía mundial, los avances tecnológicos y las migraciones tienen importantes consecuencias para la educación. El comercio internacional y la desreglamentación de las economías y los mercados de trabajo no solo han contribuido al crecimiento, sino que además han provocado cambios en las estructuras de empleo de los países adelantados, en transición y en desarrollo (Banco Mundial, 1994, p. 27).

En esta fase histórica de la investigación, lo que se dilucida es cómo funciona la calidad de la educación superior en el dispositivo discursivo episteme. El dispositivo discursivo acerca de los enunciados sobre calidad de la educación superior en Colombia, entre 1991 y 2013, tiene conexiones con otros enunciados del Plan Atcon en 1963 y la Conferencia Mundial sobre Educación 
para todos en 1990; se visibiliza para la educación, en general, desde la Declaración de Quito en 1991 y se comienza a profundizar a partir de 1998 en la Declaración Mundial sobre la Educación Superior en el Siglo XXI. Desde allí, la calidad es un pretexto para la regulación y el control de instituciones de educación superior y comunidades educativas.

\section{Conclusiones}

Los anteriores resultados en el proceso arqueológico visibilizan relaciones de enunciados coexistentes, yuxtapuestos y que interactúan por condiciones, reinscripciones, transcripciones, límites y correlaciones al interior de los discursos. Los enunciados relacionan calidad de la educación superior con funciones, propósitos curriculares, insumos, recursos económicos y físicos y talento humano; también determinan la calidad desde la medición, resultados, evaluaciones y pruebas estandarizadas; reconocen la calidad a partir del prestigio, excelencia, certificación y acreditación; y concretan la calidad y sus relaciones con el mercado y las demandas globales de fuerza de trabajo. La calidad se convierte en objeto de conocimiento para satisfacer exigencias del mercado; excluye implícitamente la ciudadanía, equidad, integración nacional y autonomía, y las supedita a los procesos de acreditación con reconocimiento.

\section{Referencias bibliográficas}

Atcon, R. P. (1963) La universidad latinoamericana. Clave para un enfoque conjunto del desarrollo coordinado social, económico y educativo en América Latina. Edición digital 2009 por Christian Hernández Amaya. Bogotá. Edición electrónica gratuita. Recuperado de: http://www.slideshare.net/paideiainvestigativa/la-universidadlatinoamericana-rudolph-atcon

Banco Mundial. Banco Internacional de Reconstrucción y Fomento (1994). El desarrollo en la práctica. La enseñanza superior. Las lecciones derivadas de la experiencia. Washington, D.C. Texto completo en: http://firgoa.usc.es/drupal/files/010-1344Sp.pdf

Banco Mundial (Banco Internacional de Reconstrucción) (1996) Prioridades y Estrategias para la Educación. Examen del Banco Mundial. Recuperado de:http://wwwwds.worldbank.org/external/default/WDSContentServer/WDSP/IB/2005/06/13/ 000160016_20050613172136/Rendered/PDF/14948010spanish.pdf

Constitución Política de Colombia (1991) Recuperado de: http://web.presidencia.gov.co/ constitucion/index.pdf

Deleuze, G. (1987). Foucault. Barcelona: Paidós

Foucault, M. (1969). La arqueología del saber. México: S. XXI.

Foucault, M. (1991). Saber y Verdad. Madrid: La Piqueta

Foucault, M. (1993). El pensamiento del afuera. Valencia: Pretextos 
Foucault, M. (1998). Historia de la sexualidad I. La voluntad de saber. Madrid: S. XXI

Foucault, M. (2003). Sobre la Ilustración. Estudio preliminar de Javier de la Higuera. Traducción de Javier de la Higuera, Eduardo Bello y Antonio Campillo. Madrid: Editorial Tecnos.

Hurtado Valero, P. M. (1994). Michel Foucault. Un proyecto de Ontología Histórica. Málaga: Librería Ágora.

Ley 30 del 28 de diciembre de 1992. "Por la cual se organiza el servicio público de la Educación Superior" Recuperado de: http://www.mineducacion.gov.co/1621/article-86437.html

Martínez Posada, J. E. (2010). La universidad productora de productores: entre biopolitica y subjetividad. Tesis Doctoral. Bogotá: Universidad de La Salle.

Morey Farré, M. (1983). Lectura de Foucault. Madrid: Taurus.

OEI (Organización de Estados Iberoamericanos para la Educación, la Ciencia y la Cultura) (1999) IX Conferencia Iberoamericana de Educación Calidad de Educación: equidad, desarrollo e integración ante el reto de la globalización. La Habana, Cuba Recuperado de: http://www.oei.es/ixcie.htm

Presidencia de la República. Plan de Desarrollo «La revolución pacífica» Plan de Desarrollo Económico y Social. (1991) Gobierno de César Gaviria (1990-1994). Bogotá: Departamento Nacional de Planeación (DNP) Recuperado de: https://www.dnp.gov.co/ PND/PlanesdeDesarrolloanteriores.aspx

Ramírez Peña, L. A. (2004). Discurso y lenguaje en la educación y la pedagogía. Bogotá: Cooperativa Editorial Magisterio

Ramírez Peña, L. A. (2008a). Comunicación y Discurso. La perspectiva polifónica en los discursos literario, cotidiano y científico. Bogotá: Magisterio

Ramírez Peña, L. A. (2008b). La calidad de la educación en un currículo postmoderno. Tesis Doctoral. Los Ángeles, California.

UNESCO. OREALC. (1990). Conferencia mundial sobre educación para todos. Marco de acción para satisfacer las necesidades básicas de aprendizaje. En: Proyecto principal de educación en América Latina y el Caribe. Boletín 21. Santiago de Chile. Recuperado de: http://unesdoc.unesco.org/images/0008/000861/086117s.pdf

UNESCO. OREALC. (1991). Declaración de Quito. En: Boletín Proyecto Principal de Educación en América Latina y el Caribe. Boletín 24. Santiago de Chile. Texto completo en: http:// unesdoc.unesco.org/images/0009/000905/090515s.pdf

UNESCO. (1998). Declaración Mundial sobre la Educación Superior en el Siglo XXI. Visión y Acción. Marco de acción prioritaria para el cambio y el desarrollo de la Educación Superior. París: Ediciones UNESCO Recuperado de: http://www.unesco.org/education/ educprog/wche/declaration_spa.htm

Williamson, John. (1990) ¿What Washington Means by Policy Reform? Chapter 2 from Latin American Adjustment: How Much Has Happened? Peterson Institute for International Economics. Washington. Recuperado de: http://www.iie.com/publications/papers/ paper.cfm?researchid $=486$

116 


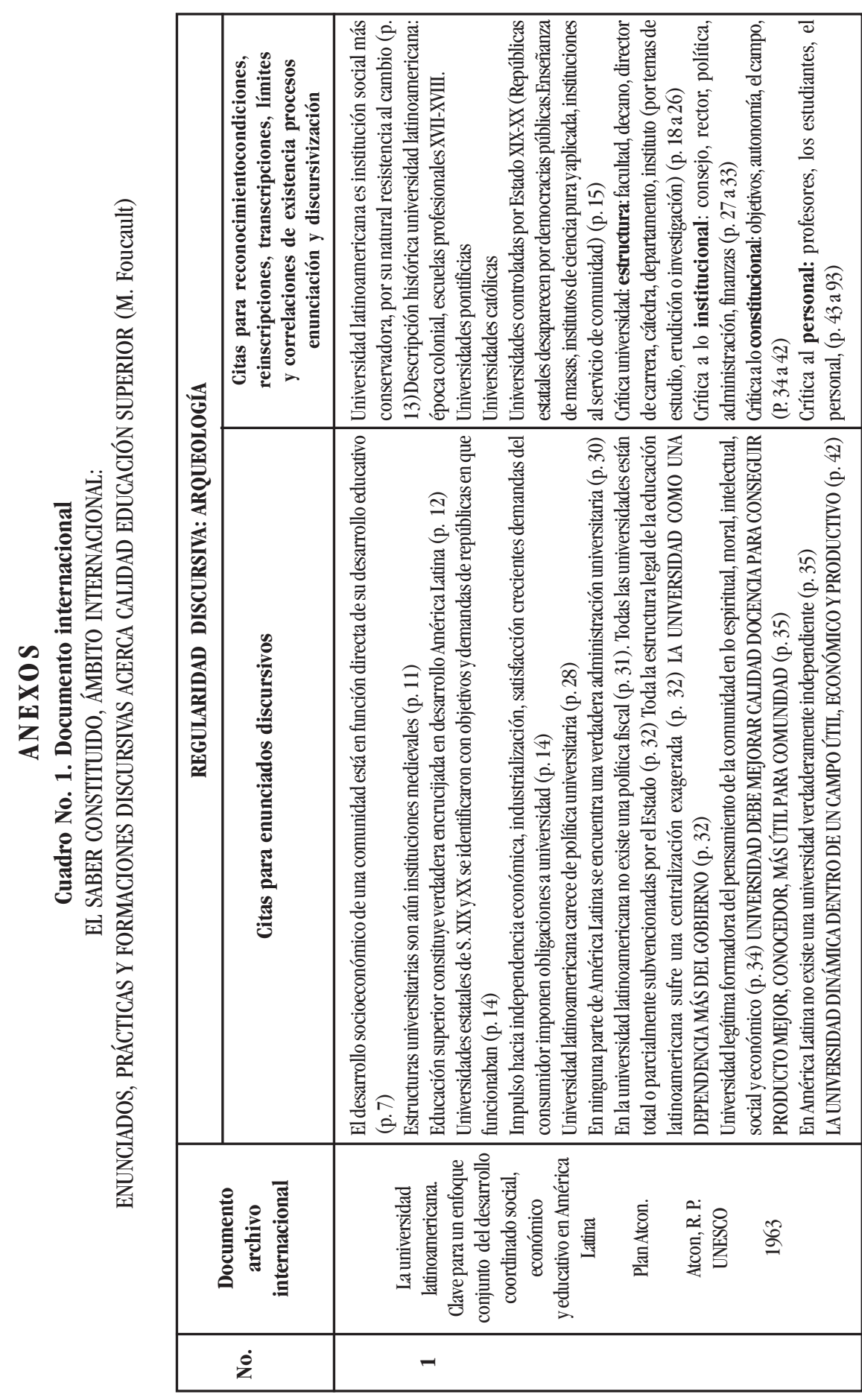




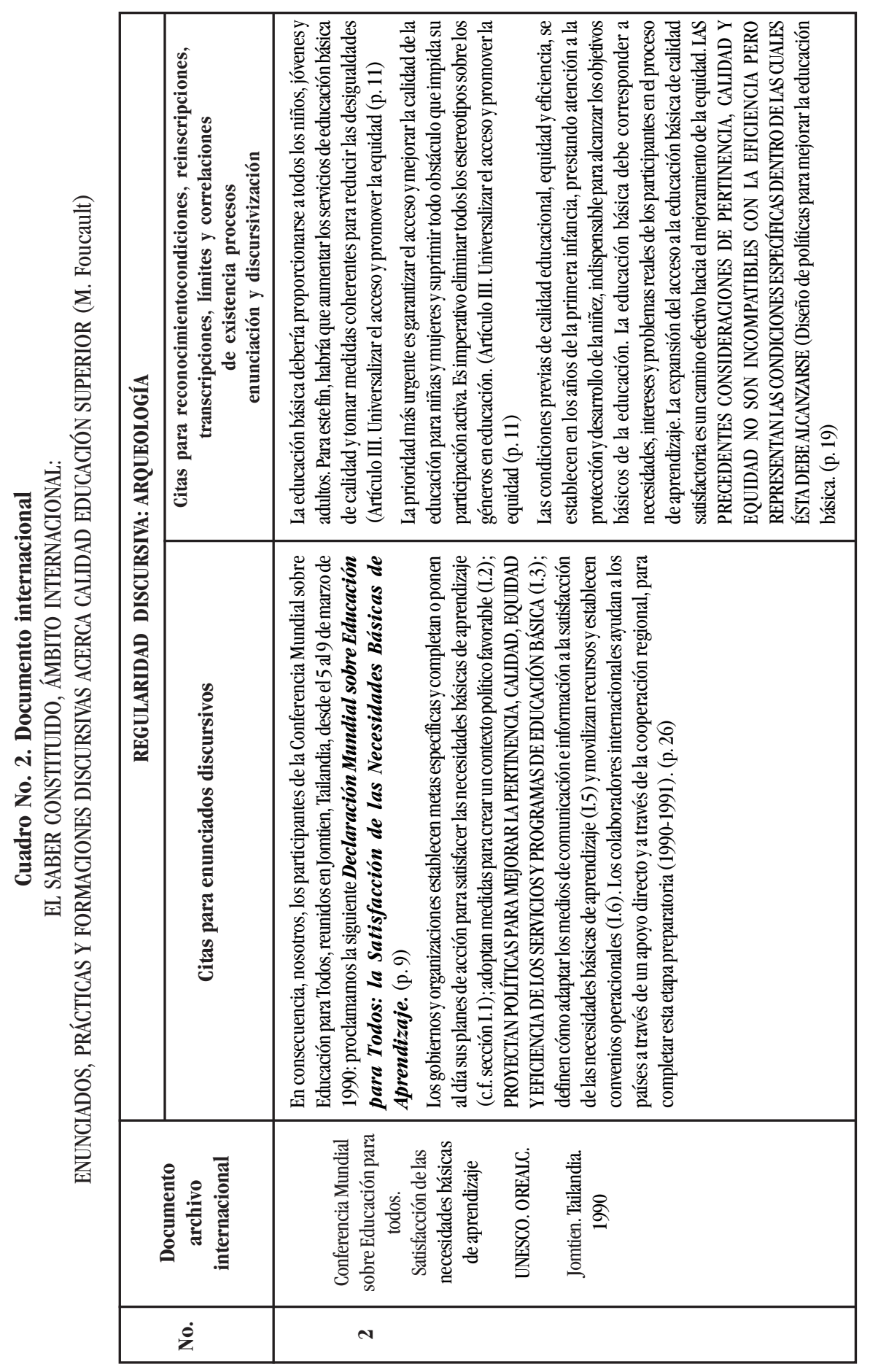

118 
Luz E. Batanelo García

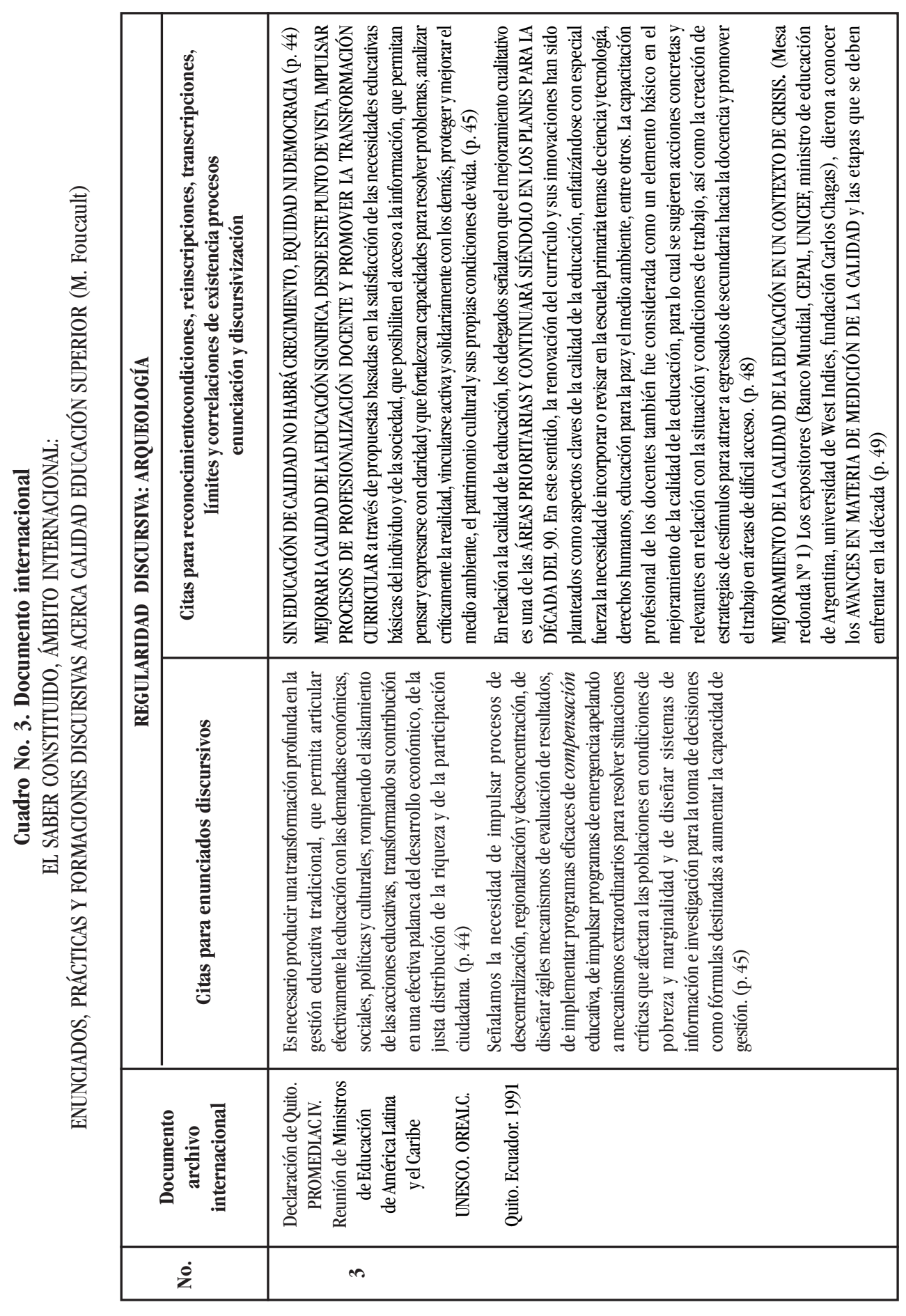

Cuadernos de Lingüística Hispánica n . 24, Julio-Diciembre 2014, pp. 105-122 


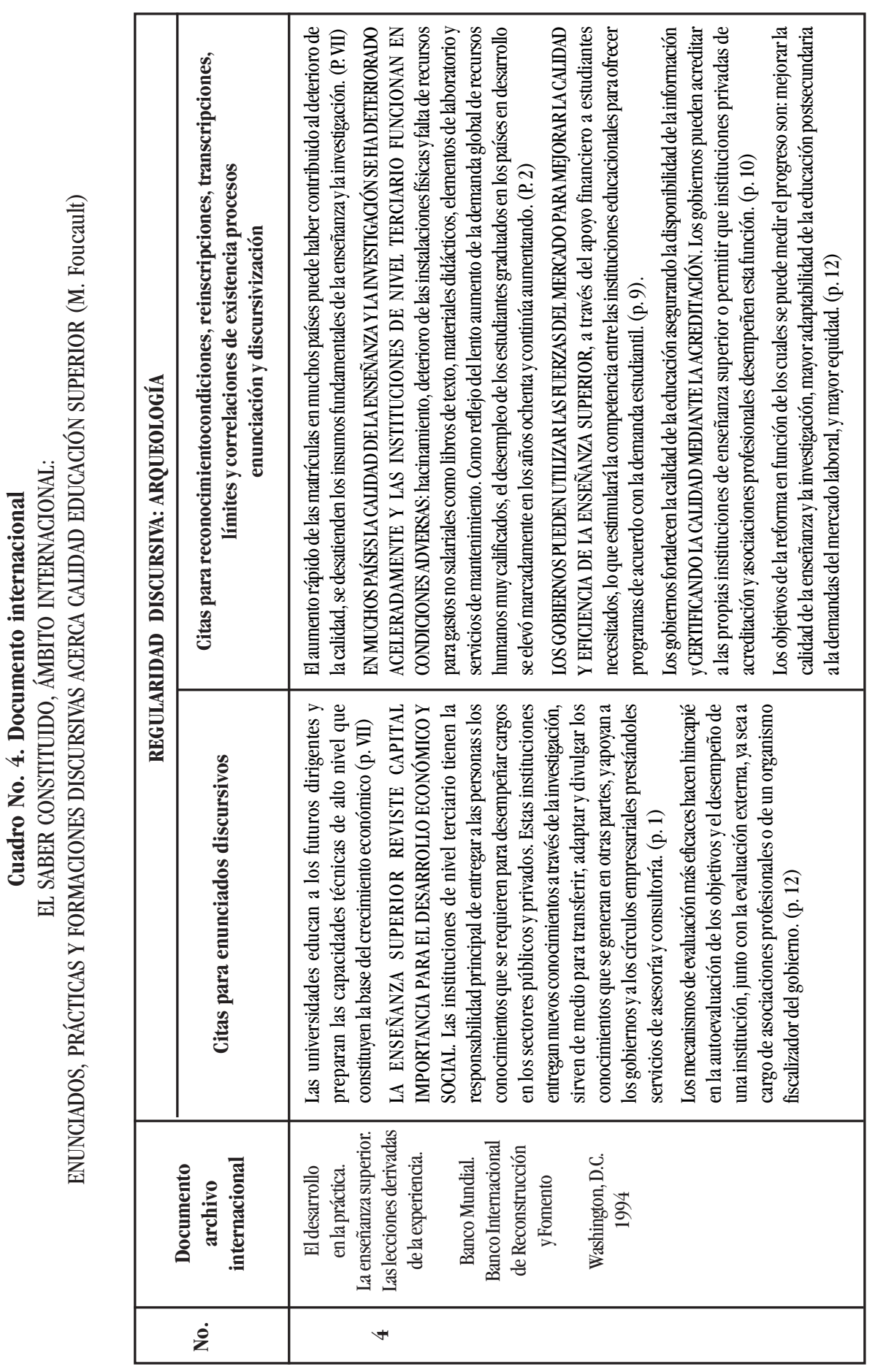


Luz Elena Batanelo García

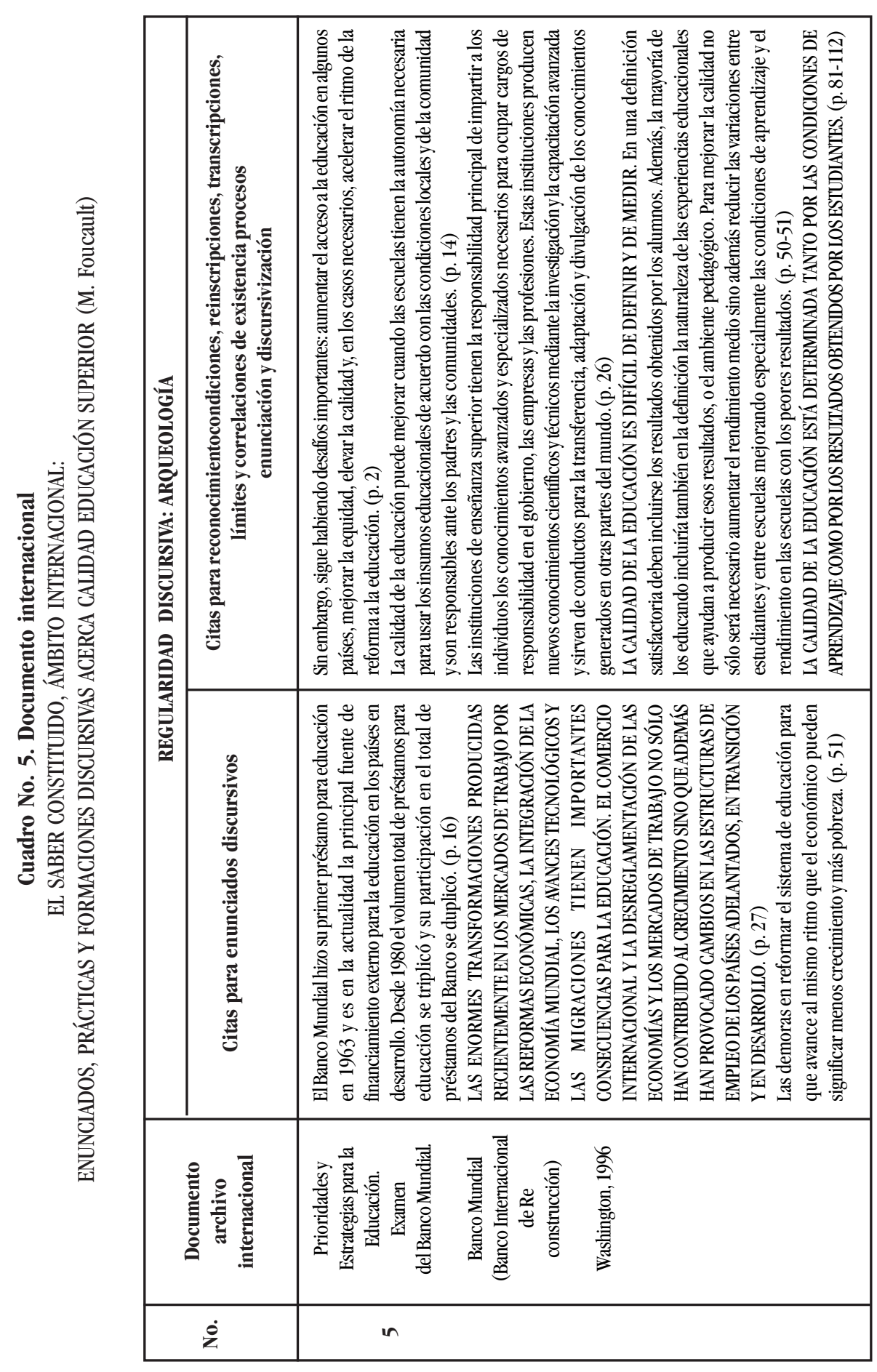

Cuadernos de Lingüística Hispánica nº. 24, Julio-Diciembre 2014, pp. 105-122 


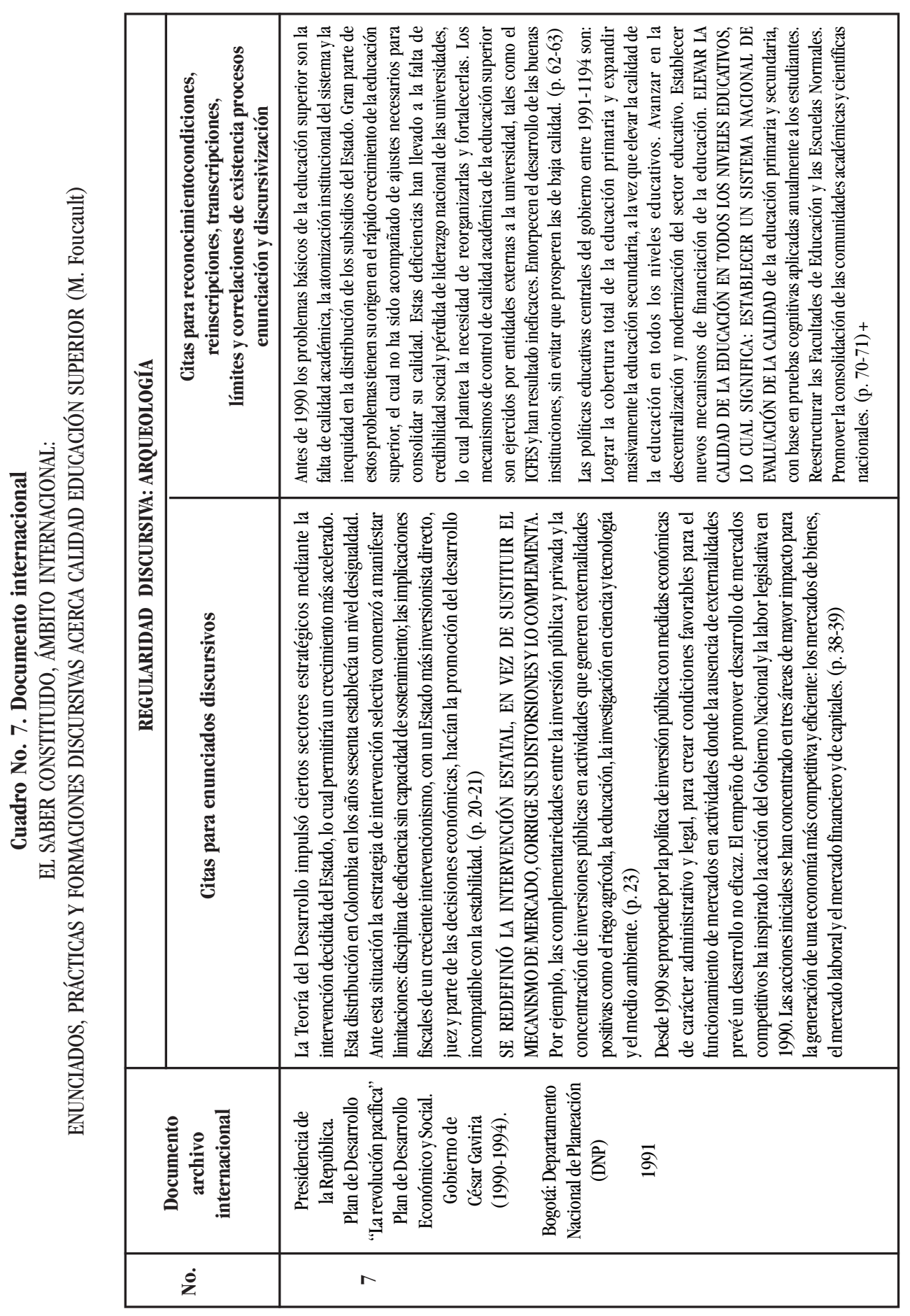




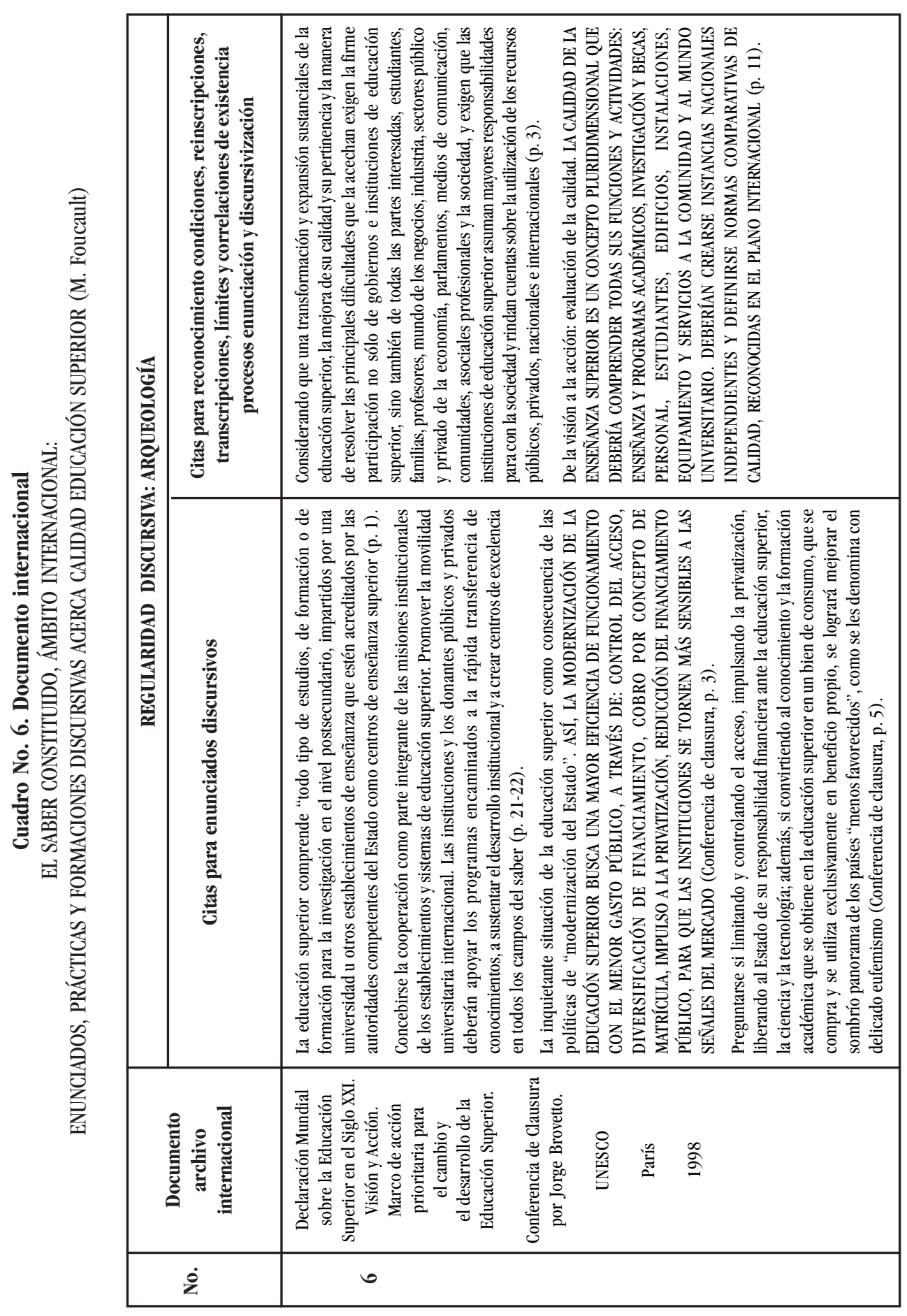

